\title{
High Energy Neutrinos from Dark Matter Decay
}

\author{
Zurab Berezhiani* ${ }^{* a b}$, Riccardo Biondi ${ }^{a, b}$ and Askhat Gazizov ${ }^{a}$ \\ a Dipartimento di Fisica e Chimica, Università di L'Aquila, 67100 Coppito, L'Aquila, Italy \\ ${ }^{b}$ INFN, Laboratori Nazionali del Gran Sasso, 67010 Assergi, L'Aquila, Italy \\ E-mail: zurab.berezhiani@aquila.infn.it
}

\begin{abstract}
Discovery of high-energy neutrino events by the IceCube Collaboration [1] opened a new era of experimental neutrino astrophysics. The analysis of starting and uncontained cascade and track events based on multiple years of IceCube data [2] leaves no doubt that the excess of the neutrino events above $100 \mathrm{TeV}$ or so cannot be explained by atmospheric neutrinos. Isotropic distribution of these events, showing no significant evidence of spatial or temporal correlations with known sources, points to extragalactic origin of high energy neutrinos. However, the production mechanism of this cosmic component is not clear yet. It might have astrophysical origin being produced by cosmic rays with a typical power law spectrum, or cosmological origin related to dark matter decay which would produce the neutrino spectrum in a form of one ore more bumps. Here we present a model of decaying dark matter represented by heavy mirror neutrinos, with masses of few PeV, from a parallel gauge sector with very large electroweak scale, in which ordinary high energy neutrinos are produced via the majoron portal. In this case the neutrino spectrum would consist of two bumps, one with maximal energy of about $0.5 \mathrm{PeV}$, and another with maximal energy of about $5 \mathrm{PeV}$. In addition, the majoron decay produces the neutrino mass eigenstates $v_{1,2,3}$ with fractions proportional to the neutrino mass squared, $F_{1}: F_{2}: F_{3}=m_{1}^{2}: m_{2}^{2}: m_{3}^{2}$, with specific implications for the flavor composition of the IceCube neutrinos. Thus, precise determination of the energy spectrum and flavor content of the IceCube neutrinos in the future can discriminate between their cosmological and astrophysical origins.
\end{abstract}

Neutrino Oscillation Workshop

4 - 11 September, 2016

Otranto (Lecce, Italy)

\footnotetext{
* Speaker.
} 
The astrophysical high energy (HE) neutrinos can be originated via $\pi, K$ decays produced by collisions of cosmic rays to background gas or light at their acceleration sites. This component is expected to have a typical power law energy spectrum $\phi(E) \propto E^{-\gamma}$ with $\gamma=2$ or so, and its original composition, in terms of of flavor eigenstates $v_{e}, v_{\mu}$ and $v_{\tau}$, should obey the proportion $\tilde{f}_{e}: \tilde{f}_{\mu}: \tilde{f}_{\tau} \simeq 1 / 3: 2 / 3: 0$. However, due to large angles of the neutrino mixing, the neutrino oscillations at cosmological distances modify the initial composition into nearly democratic blend of flavors $f_{e}: f_{\mu}: f_{\tau} \simeq 1 / 3: 1 / 3: 1 / 3$ detectable at the Earth.

The power law description of cosmic neutrino energy spectrum evidences certain tensions. Namely, according to paper 1081 of Ref. [2], HE starting events indicate towards a soft power law spectrum per neutrino flavor $\phi(E)=\phi_{0} \times(E / 100 \mathrm{TeV})^{-\gamma}$, with $\gamma=2.58 \pm 0.25$ and $\phi_{0}=2.2 \pm$ 0.7 (in units of $10^{-18} \mathrm{GeV}^{-1} \mathrm{~cm}^{-2} \mathrm{sr}^{-1} \mathrm{~s}^{-1}$ ). The independent analysis of uncontained showers starting near the edge of the detection volume show even softer spectrum: fits of two samples corresponding to arrival directions from north and south hemispheres are well consistent and lead to $\gamma^{\text {north }}=2.69 \pm 0.34$ and $\phi_{0}^{\text {north }}=1.7 \pm 1.3$ against $\gamma^{\text {south }}=2.68 \pm 0.22$ and $\phi_{0}^{\text {south }}=1.9 \pm 0.8$, see paper 1109 of [2]. On the other hand, analysis of down-going muon events directed from the northern hemisphere, paper 1079 of [2], indicate towards much harder spectrum of cosmic $v_{\mu}$ component, with $\gamma=1.9 \pm 0.2$ and $\phi_{0}=0.66 \pm 0.40$. In addition, HE neutrino events observed by IceCube have interesting spectral features. Namely, there is an evident deficit of events in the range between $0.5 \mathrm{PeV}$ and $1 \mathrm{PeV}$, indicated by the data of both starting and uncontained cascade events as well as by downgoing muon tracks from northern hemisphere [2]. Such a gap in the energy spectrum is difficult to explain in models of HE neutrinos of astrophysical origin. In addition, the spectrum is apparently cut off at energies larger than few $\mathrm{PeV}$, as indicated by non-observation of cascade events due to the Glashow resonance $\bar{v}_{e}+e^{-} \rightarrow W^{-} \rightarrow$ hadrons at $E_{\text {res }} \simeq 6 \mathrm{PeV}$.

Alternatively, cosmic HE neutrinos can be produced by decaying dark matter (DDM) particles with masses of few PeV, as e.g. in Refs. [3, 4]. In this case their energy spectrum is not expected to be a power-like; it can have a form of bumps and it cannot extend to very high or very low energies. Interestingly, the DDM scenario has independent motivation. As it was shown in Ref. [5], the presence of DDM component, constituting a fraction of few per cent of the overall density of dark matter at the recombination epoch and then decaying in some invisible channels before the present epoch, can alleviate discrepancies between the values of cosmological parameters as are the Hubble constant $H_{0}$, dark energy fraction $\Omega_{\Lambda}$, and amplitude of matter fluctuations $\sigma_{8}$, deduced from the precision measurements of the CMB anisotropies by the Planck collaboration on the one side, and obtained from the direct astronomical measurements at low redshifts on the other side.

Here we consider a prototype model, which can provide a needed fraction and lifetimes of DDM particles, and can reproduce the observed spectrum of the cosmic HE neutrinos. We suppose that dark matter emerges from a parallel mirror sector, a hidden gauge sector with the Lagrangian similar to that of the ordinary particles (for reviews, see e.g. [6]). Our model is based on two copies of the Standard Model, $S U(3) \times S U(2) \times U(1)$ and $S U(3)^{\prime} \times S U(2)^{\prime} \times U(1)^{\prime}$, the first describing ordinary particles and another their mirror partners. However, in the spirit of Refs. [7], we assume that the mirror electroweak scale $\left\langle\phi^{\prime}\right\rangle=V^{\prime}$ is much larger than the ordinary one $\langle\phi\rangle=V=174$ $\mathrm{GeV}$, where $\phi$ and $\phi^{\prime}$ are ordinary and mirror Higgs doublets.

Let us assume now that two sectors have a common lepton number symmetry $U(1)_{L}$ and the neutrino Majorana masses in both worlds are induced due to its spontaneous breaking by the VEV 
of scalar $\chi$ with $L=-2,\langle\chi\rangle=V_{\chi}$, via higher order operators

$$
\begin{aligned}
& \frac{y_{\alpha \beta} \chi}{\mathscr{M}^{2}}\left(\phi l_{\alpha}\right)\left(\phi l_{\beta}\right) \Rightarrow m_{i}=\frac{y_{i} V^{2} V_{\chi}}{\mathscr{M}^{2}}=y_{i}\left(\frac{10^{11} \mathrm{GeV}}{\mathscr{M}}\right)^{2}\left(\frac{V_{\chi}}{10^{7} \mathrm{GeV}}\right) \times 0.03 \mathrm{eV}, \\
& \frac{y_{\alpha \beta}^{\prime} \chi}{\mathscr{M}^{\prime 2}}\left(\phi^{\prime} l_{\alpha}^{\prime}\right)\left(\phi^{\prime} l_{\beta}^{\prime}\right) \Rightarrow M_{i}=\frac{y_{i}^{\prime} V^{\prime 2} V_{\chi}}{\mathscr{M}^{\prime 2}}=y_{i}^{\prime}\left(\frac{V^{\prime}}{\mathscr{M}^{\prime}}\right)^{2}\left(\frac{V_{\chi}}{10^{7} \mathrm{GeV}}\right) \times 10^{7} \mathrm{GeV}
\end{aligned}
$$

where $l_{\alpha}=(v, e)_{\alpha}$ and $l_{\alpha}^{\prime}=\left(v^{\prime}, e^{\prime}\right)_{\alpha}$ respectively are the standard and mirror lepton doublets, $y_{\alpha \beta}, y_{\alpha \beta}^{\prime}$ are (symmetric) matrices of the coupling constants, with $\alpha, \beta=e, \mu, \tau$ being the lepton flavor indices, $y_{i}, y_{i}^{\prime}, i=1,2,3$, are their eigenvalues, and $\mathscr{M}$ and $\mathscr{M}^{\prime}$ are some large cutoff scales. (Operators (1) can be obtained in the context of renormalizable theories as discussed in [8].) Therefore, if we take $V^{\prime} \leq \mathscr{M}^{\prime}$ and $V_{\chi} \sim 10^{7} \mathrm{GeV}$, then mirror neutrino masses can be of few $\mathrm{PeV}, M_{i} \sim V_{\chi}$. Let us order them for definiteness as $M_{1}<M_{2}<M_{3}$, taking their benchmark values as $M_{3}=10 \mathrm{PeV}, M_{2}=4 \mathrm{PeV}$ and $M_{1}=3.5 \mathrm{PeV}$.

Mirror neutrinos $v_{i}^{\prime}$ can be produced non-thermally in the early Universe, with initial abundances $n_{i}$ which are not necessarily equal. Should $v_{3}^{\prime}$ and $v_{2}^{\prime}$ be stable, their fractions in dark matter would be given by $n_{3} M_{3}=f_{3} \rho_{d m}$ and $n_{2} M_{2}=f_{2} \rho_{d m}$. However, for realizing the DDM scenario [5], we assume that heavier species $v_{3}^{\prime}$ and $v_{2}^{\prime}$ are unstable, with decay times somewhat less than the age of the Universe, $\tau_{3}, \tau_{2}<t_{u}=14 \mathrm{Gyr}$, while their initial fractions $f_{3}$ and $f_{2}$ are $\sim 10^{-2}$.

These species can decay radiatively with mirror photon emission. It is well known that for ordinary neutrinos the rate of radiative decay $v_{i} \rightarrow v_{j}+\gamma, m_{i}>m_{j}$, is extremely small [9]:

$$
\Gamma\left(v_{i} \rightarrow v_{j}+\gamma\right)=\frac{9 \alpha G_{F}^{2} m_{i}^{5}}{4(4 \pi)^{4}}\left(\frac{m_{\tau}}{M_{W}}\right)^{4}\left|V_{i \tau}^{*} V_{j \tau}\right|^{2} \sim\left(\frac{m_{i}}{0.1 \mathrm{eV}}\right)^{5} \times 10^{-41} \mathrm{yr}^{-1}
$$

However, mirror neutrinos $v_{2,3}^{\prime}$ have masses of few PeV, while the mirror Fermi constant rescales as $G_{F}^{\prime} \sim G_{F}\left(V / V^{\prime}\right)^{2}$ [7]. Hence, for $V^{\prime} \sim 10^{15} \mathrm{GeV}$ their radiative decay times can be of few Gyr:

$$
\Gamma\left(v_{i}^{\prime} \rightarrow v_{j}^{\prime}+\gamma^{\prime}\right) \sim\left(\frac{M_{i}}{10 \mathrm{PeV}}\right)^{5}\left(\frac{10^{13} V}{V^{\prime}}\right)^{4} \times 10^{-10} \mathrm{yr}^{-1}
$$

Spontaneous breaking of global $U(1)_{L}$ brings into the game a Goldstone boson, coined as Majoron [10]. The majoron $\eta$ is diagonally coupled with the mass eigenstates $v_{1,2,3}$ and $v_{1,2,3}^{\prime}$, respectively with the coupling constants $g_{\eta i}=m_{i} / f_{L}$ and $g_{\eta i}^{\prime}=M_{i} / f_{L}$, where $f_{L}=\sqrt{2} V_{\chi}$ (c.f. (1)), and thus the decays $v_{i}^{\prime} \rightarrow v_{j}^{\prime}+\eta$ cannot occur. In addition, the massless majoron cannot decay into neutrinos.

However, one can include Planck scale induced operators [11]. In particular, operators like $\frac{\left(\chi^{\dagger} \chi\right) \chi}{M_{P l}^{4}}\left(\phi^{\prime} l_{\alpha}^{\prime}\right)\left(\phi^{\prime} l_{\beta}^{\prime}\right)$ induce the majoron couplings non-diagonal between the mass eigenstates $v_{1,2,3}^{\prime}$, with the tiny constants $g_{\eta i j}^{\prime} \sim V_{\chi}^{2} V^{\prime 2} / M_{P l}^{4} \sim 10^{-30} \times\left(V_{\chi} / 10^{7} \mathrm{GeV}\right)^{2}$. Now the decays with the majoron emission $v_{i}^{\prime} \rightarrow v_{j}^{\prime}+\eta$ become possible, however with tiny branching ratios with respect to main invisible decay channel (3). On the other hand, operators as $\frac{1}{M_{P l}}\left(\chi^{\dagger} \chi\right)^{2}\left(\chi+\chi^{\dagger}\right)$, etc. transform the majoron into a pseudo-Goldstone particle inducing its mass $m_{\eta} \sim\left(V_{\chi}^{3} / M_{P l}\right)^{1 / 2} \sim$ $\left(V_{\chi} / 10^{7} \mathrm{GeV}\right)^{3 / 2} \times 10 \mathrm{GeV}$ [11]. Hence, the relativistic majorons with $\mathrm{PeV}$ energies, produced in the decays of $v_{3}^{\prime} \rightarrow v_{2}^{\prime}+\eta$, in itself decay in two neutrinos in time scale much shorter than $t_{u}$ : $\Gamma(\eta \rightarrow 2 v) \simeq\left(g_{\eta i}^{2} / 8 \pi\right)\left(m_{\eta}^{2} / E_{\eta}\right)$. As far as $g_{\eta i}=m_{i} / f_{L}, m_{\eta}^{2} \sim f_{L}^{3} / M_{P l}$ and $E_{\eta} \simeq f_{L} / 2$, we obtain 


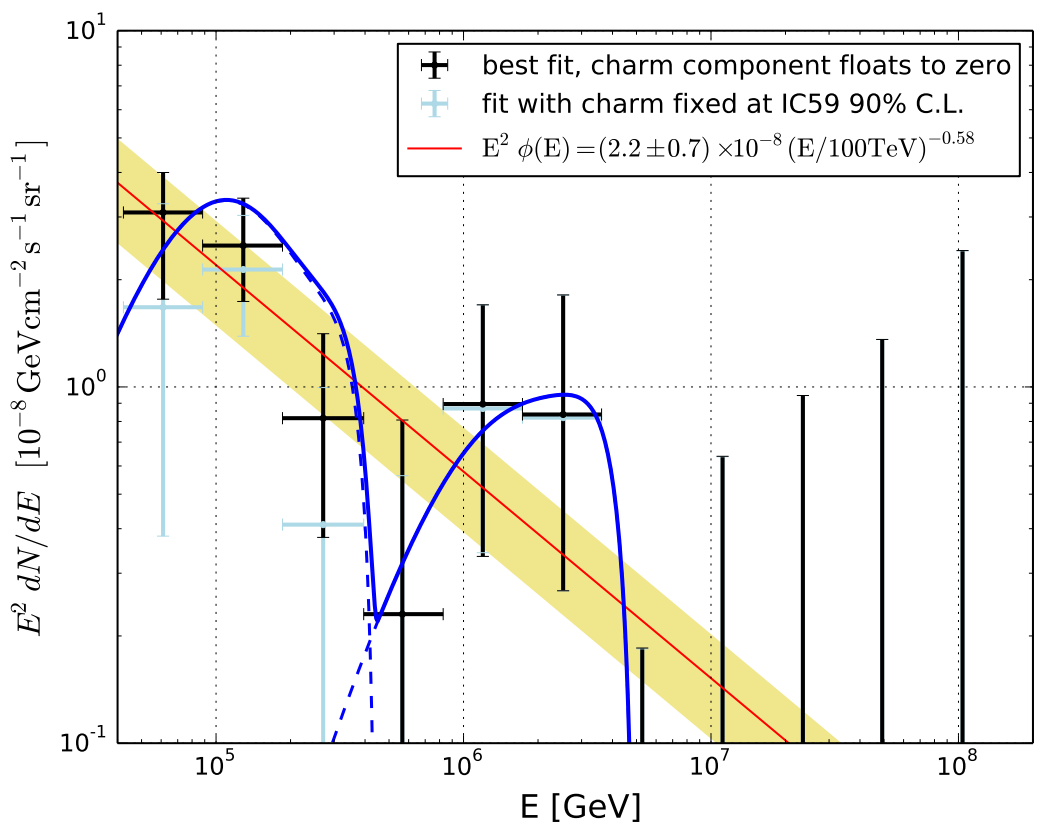

Figure 1: Neutrino spectrum in our DDM model (blue solid) vs. best fit power-law fit (red) of the IceCube neutrino fluxes in different energy bins reported in Ref. [2].

$\Gamma(\eta \rightarrow 2 v) \simeq 0.1 m_{i}^{2} / M_{P l} \geq 10^{-9} \mathrm{yr}^{-1}$, where we have taken into account that the mass of heaviest neutrino should be $\geq 0.05 \mathrm{eV}$.

The decay $v_{i}^{\prime} \rightarrow v_{j}^{\prime}+\eta$ at rest produces mono-energetic majorons with $E_{i j}=\frac{1}{2}\left(M_{i}^{2}-M_{j}^{2}\right) / M_{i}$, which then decay in flight, $\eta \rightarrow 2 v$, producing the neutrinos with flat spectrum abruptly cut at maximal energy $E_{\max }=E_{i j}$. Generically, the flux of cosmic neutrinos produced in this way consists of two components, one originated via dark matter decay in the Galaxy halo and having the above flat spectrum, and another originated via dark matter decay in whole Universe. For $\tau_{2,3} \ll t_{u}$, the most of extra-galactic neutrinos are produced at higher redshifts which degrades their spectrum towards lower energies, and, in addition, the hard galactic component can be neglected. Then for extragalactic neutrino fluxes produced via the decays $v_{i}^{\prime} \rightarrow v_{j}^{\prime}+\eta$ one obtains

$$
\Phi_{31}^{v}=2 B_{31} f_{3} \Phi_{0}, \quad \Phi_{32}^{v}=2 B_{32} f_{3} \Phi_{0}, \quad \phi_{21}^{v}=2 B_{21}\left(\frac{M_{3}}{M_{2}} f_{2}+f_{3} B_{32}\right) \Phi_{0}
$$

where $\Phi_{0}=(c / 4 \pi) \times\left(\Omega_{d m} \rho_{\mathrm{cr}} / M_{3}\right)=3 \times 10^{-4} f_{X}\left(10 \mathrm{PeV} / M_{3}\right) \mathrm{cm}^{-2} \mathrm{~s}^{-1} \mathrm{sr}^{-1}$ and $B_{j i}$ are the branching ratios of $v_{i}^{\prime} \rightarrow v_{j}^{\prime}+\eta$ decays. Taking our benchmark values for $M_{i}$, the decays $v_{3}^{\prime} \rightarrow v_{1,2}^{\prime}$ should produce the neutrinos respectively with $E_{\max }=4.2 \mathrm{PeV}$ and $4.4 \mathrm{PeV}$, while decay $v_{2}^{\prime} \rightarrow v_{1}$ would produce lower energy neutrinos with $E_{\max }=0.47 \mathrm{PeV}$. The corresponding bumpy spectrum of HE neutrinos is presented on Fig. 1 where we have also taken $\tau_{2,3}=t_{u} / 3$.

The study of flavor composition of the IceCube neutrino events can also give useful hints for discriminating the origin of this cosmic component. Dark matter decay into the neutrinos generically produces the neutrino mass eigenstates $v_{1,2,3}$, in a model-dependent proportion $F_{1}$ : 


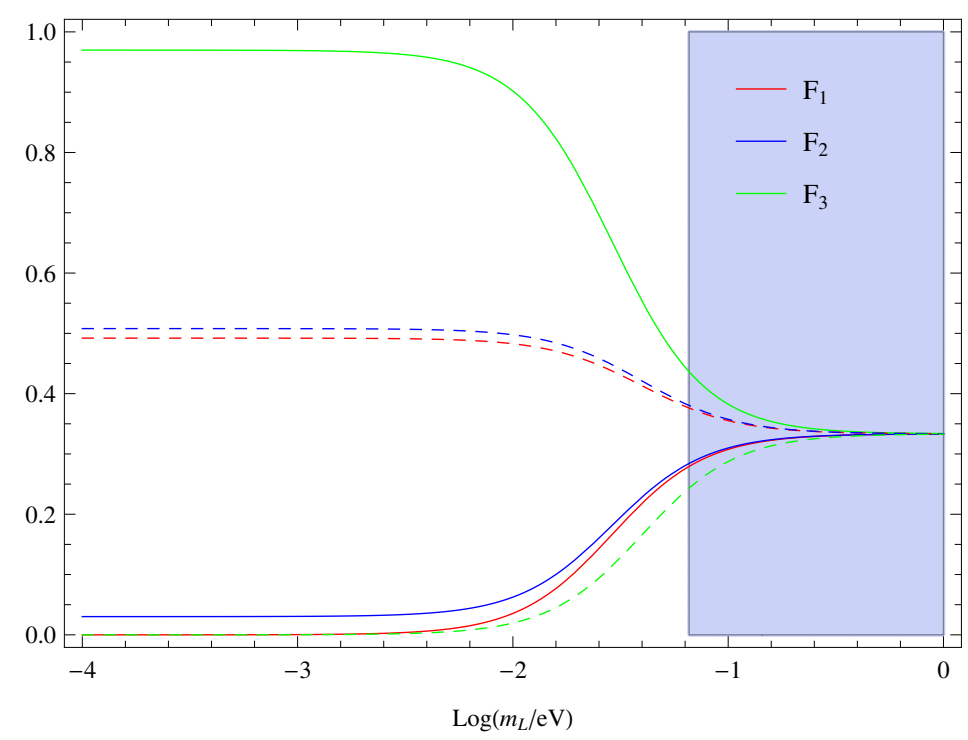

Figure 2: Fractions $F_{1,2,3}$ as functions of the lightest neutrino mass, $m_{1}$ in NH model (solid) and $m_{3}$ in IH model (dash). The shaded area is excluded by cosmological limit on the neutrino masses $m_{1}+m_{2}+m_{3}<$ $0.32 \mathrm{eV}$.

$F_{2}: F_{3}$. In particular, in the context of our model the decays $\eta \rightarrow 2 v$ of the majoron coupled to neutrinos with $g_{\eta i}=m_{i} / f_{L}$, produces the mass eigenstates $v_{1,2,3}$ in a proportion $F_{1}: F_{2}: F_{3}=m_{1}^{2}$ : $m_{2}^{2}: m_{3}^{2}$. Therefore, for the normal hierarchy (NH) of neutrino masses $m_{3}>m_{2}>m_{1}$ we expect that dominantly $v_{3}$ is produced, while in the case of inverse hierarchy (IH), $m_{1} \approx m_{2}>m_{3}$, dominant contribution is given by an equal blend of $v_{1}$ and $v_{2}$ (see Fig. 2). However, in the context of realistic models based on flavor symmetry [12], the majoron can be the same particle as familon having nondiagonal couplings between different neutrinos, directly or inversely reflecting the neutrino mass hierarchy. In any case, the DDM scenario produces the neutrinos in some combination of mass eigenstates and its predictions for the flavor composition of IceCube neutrinos are different from that of astrophysical neutrinos produced by standard interactions in some combinations of flavor eigenstates. The possible final flavor compositions for the astrophysical neutrinos and for the DDM neutrinos are shown on Fig. 3. As we see, the latter case provides much wider possibilities.

Neutrino flavor (or weak interaction) eigenstates $v_{\alpha}(\alpha=e, \mu, \tau)$ are superpositions of the neutrino mass eigenstates $v_{i}(i=1,2,3) ; v_{\alpha}=\sum_{i} V_{\alpha i} v_{i}$, where $V_{\alpha i}$ is the neutrino mixing matrix. The mass eigenstates do not oscillate and remain the same during propagation. The probability to detect mass eigenstate $v_{i}$ as a flavor state $v_{\alpha}$ is $\left|V_{\alpha i}\right|^{2}$. Therefore, a mass composition $F=$ $\left(F_{1}, F_{2}, F_{3}\right)$ corresponds to final flavor composition $f_{\alpha}=\sum_{i}\left|V_{\alpha i}\right|^{2} F_{i}$, or in explicit form

$$
\left(\begin{array}{c}
f_{e} \\
f_{\mu} \\
f_{\tau}
\end{array}\right)=\left(\begin{array}{ccc}
\left|V_{e 1}\right|^{2} & \left|V_{e 2}\right|^{2} & \left|V_{e 3}\right|^{2} \\
\left|V_{\mu 1}\right|^{2} & \left|V_{\mu 2}\right|^{2} & \left|V_{\mu 3}\right|^{2} \\
\left|V_{\tau 1}\right|^{2} & \left|V_{\tau 2}\right|^{2} & \left|V_{\tau 3}\right|^{2}
\end{array}\right)\left(\begin{array}{l}
F_{1} \\
F_{2} \\
F_{3}
\end{array}\right)=\left(\begin{array}{lll}
0.68 & 0.30 & 0.02 \\
0.15 & 0.42 & 0.43 \\
0.17 & 0.28 & 0.55
\end{array}\right)\left(\begin{array}{c}
F_{1} \\
F_{2} \\
F_{3}
\end{array}\right)
$$

where we substituted the best fit values of the neutrino mixing parameters, three angles and CP phase, according to global data analysis of Ref. [13]. The triangle shown on left panel of Fig. 


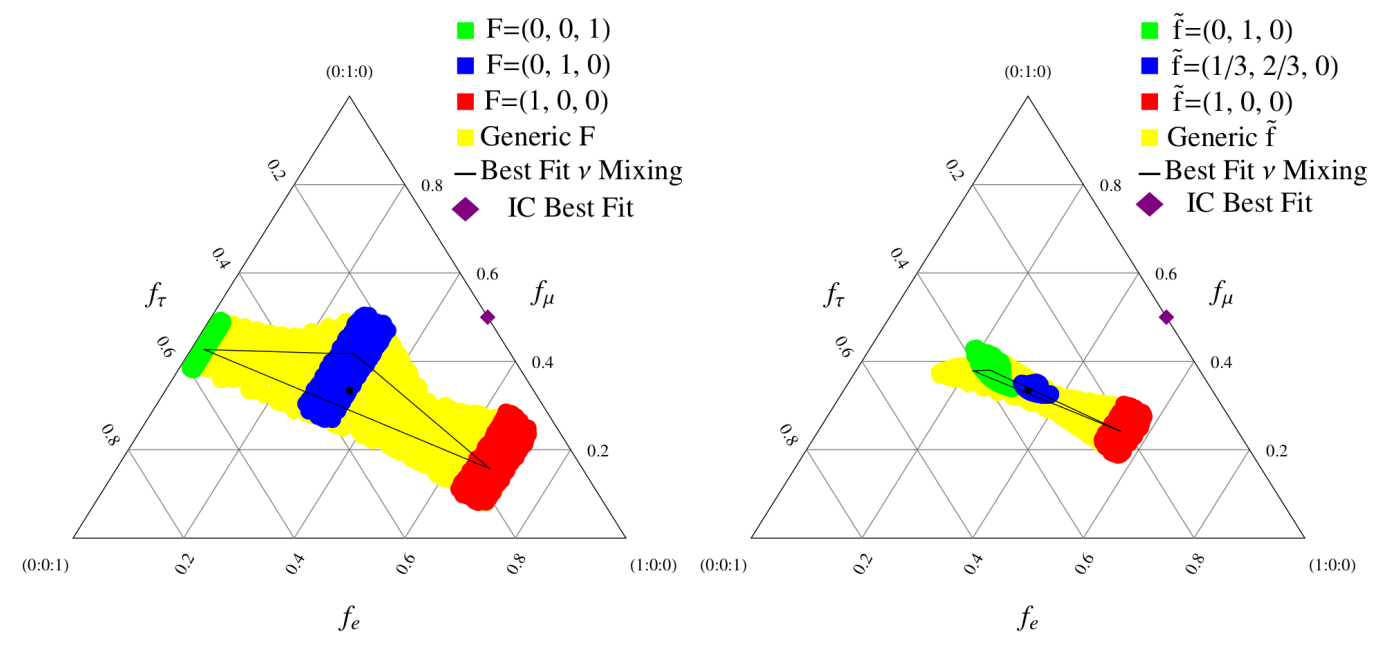

Figure 3: Left: final flavor content of cosmological neutrinos for generic initial composition of mass eigenstates $F=\left(F_{1}, F_{2}, F_{3}\right)$ and for some specific choices, predicted within $2 \sigma$ uncertainties for the neutrino mixing parameters. The triangle confines the final composition in the case when the mixing parameters are taken at their best fit numerical values. Right: the same for astrophysical neutrinos with initial flavor composition of generic form $\tilde{f}=\left(\tilde{f}_{e}, \tilde{f}_{\mu}, \tilde{f}_{\tau}\right)$ and for some specific flavor compositions.

3 confines the final flavor composition for the above numerical values of $\left|V_{\alpha i}\right|^{2}$, while the extended yellow area correspond to possible flavor content when neutrino mixing parameters are varied within $2 \sigma$ uncertainties. In a particular case when all three mass eigenstates are produced democratically, e.g. via kinetic mixing of massive dark bosons with $Z$ boson, one would have $F_{1}: F_{2}: F_{3} \simeq 1 / 3: 1 / 3: 1 / 3$ and so, in consequence of unitarity, $f_{e}: f_{\mu}: f_{\tau} \simeq 1 / 3: 1 / 3: 1 / 3$. However, in our model with the majoron mediated decays this democratic possibility is practically excluded by the cosmological upper limit on the neutrino masses, see Fig. 2.

For comparison, astrophysical neutrinos are produced by standard weak interactions in flavor eigenstates with some initial composition $\tilde{f}=\left(\tilde{f}_{e}, \tilde{f}_{\mu}, \tilde{f}_{\tau}\right)$ which by neutrino oscillations averaged at large distances is transformed to the final flavor blend $f=\left(f_{e}: f_{\mu}: f_{\tau}\right)$ detectable at the Earth, $f_{\alpha}=\sum_{\beta} P_{\alpha \beta} \tilde{f}_{\beta}$, where $P_{\alpha \beta}=\sum_{i}\left|V_{\alpha i}\right|^{2}\left|V_{\beta i}\right|^{2}$, or explicitly

$$
\left(\begin{array}{c}
f_{e} \\
f_{\mu} \\
f_{\tau}
\end{array}\right)=\left(\begin{array}{lll}
P_{e e} & P_{e \mu} & P_{e \tau} \\
P_{\mu e} & P_{\mu \mu} & P_{\mu \tau} \\
P_{\tau e} & P_{\tau \mu} & P_{\tau \tau}
\end{array}\right)\left(\begin{array}{c}
\tilde{f}_{e} \\
\tilde{f}_{\mu} \\
\tilde{f}_{\tau}
\end{array}\right)=\left(\begin{array}{ccc}
0.55 & 0.24 & 0.21 \\
0.24 & 0.38 & 0.38 \\
0.21 & 0.38 & 0.41
\end{array}\right)\left(\begin{array}{c}
\tilde{f}_{e} \\
\tilde{f}_{\mu} \\
\tilde{f}_{\tau}
\end{array}\right)
$$

where numerical values correspond to best fit values for neutrino mixing parameters taken from Ref. [13]. Thin triangle on right panel of Fig. 3, almost degenerate into a line, confines the final composition for the above numerical values of $P_{\alpha \beta}$.

Concluding, the DDM scenarios for the cosmic HE neutrinos can be discriminated with increasing event statistics at the IceCube by their spectral shape and by their flavor content.

The work was partially supported by MIUR grant PRIN 2012CPPYP7 on Astroparticle Physics. 


\section{References}

[1] M. G. Aartsen et al. [IceCube Collaboration], Phys. Rev. Lett. 111, 021103 (2013); Science 342, no. 6161, 1242856 (2013); Phys. Rev. Lett. 113, 101101 (2014).

[2] M. G. Aartsen et al. [IceCube Collaboration], arXiv:1510.05223 [astro-ph.HE].

[3] A. Esmaili, S. K. Kang and P. D. Serpico, JCAP 1412, no. 12, 054 (2014).

[4] Z. Berezhiani, Nucl. Part. Phys. Proc. 265-266, 303 (2015); R. Biondi, Nuovo Cim. C 038, 31 (2015).

[5] Z. Berezhiani, A. D. Dolgov and I. I. Tkachev, Phys. Rev. D 92, no. 6, 061303 (2015).

[6] Z. Berezhiani, Int. J. Mod. Phys. A 19, 3775 (2004); hep-ph/0508233; Eur. Phys. J. ST 163, 271 (2008).

[7] Z. Berezhiani and R. N. Mohapatra, Phys. Rev. D 52, 6607 (1995); Z. Berezhiani, A. D. Dolgov and R. N. Mohapatra, Phys. Lett. B 375, 26 (1996); Z. Berezhiani, Acta Phys. Polon. B 27, 1503 (1996); Z. Berezhiani, L. Gianfagna and M. Giannotti, Phys. Lett. B 500, 286 (2001).

[8] Z. Berezhiani, A. Y. Smirnov and J. W. F. Valle, Phys. Lett. B 291, 99 (1992); see also Z. Berezhiani, Eur. Phys. J. C 76, no. 12, 705 (2016).

[9] P. B. Pal and L. Wolfenstein, Phys. Rev. D25, 766 (1982).

[10] Y. Chikashige, R. N. Mohapatra and R. D. Peccei, Phys. Lett. B 98, 265 (1981).

[11] E. K. Akhmedov, Z. Berezhiani, R. N. Mohapatra and G. Senjanovic, Phys. Lett. B 299, 90 (1993); E. K. Akhmedov, Z. Berezhiani and G. Senjanovic, Phys. Rev. Lett. 69, 3013 (1992).

[12] Z.. Berezhiani, Phys. Lett. B 129, 99 (1983); Phys. Lett. B 150, 177 (1985); Z. Berezhiani and M. Y. Khlopov, Z. Phys. C 49, 73 (1991); Sov. J. Nucl. Phys. 51, 739 (1990) [Yad. Fiz. 51, 1157 (1990)]; Sov. J. Nucl. Phys. 52, 60 (1990) [Yad. Fiz. 52, 96 (1990)].

[13] A. Marrone, F. Capozzi, E. Lisi, D. Montanino and A. Palazzo, J. Phys. Conf. Ser. 718, no. 6, 062042 (2016). 